\title{
Neural Activity-Dependent Regulation of Radial Glial Filopodial Motility Is Mediated by Glial cGMP-Dependent Protein Kinase 1 and Contributes to Synapse Maturation in the Developing Visual System
}

\author{
๑Mari Sild, ${ }^{1,2}$ Marion R. Van Horn, ${ }^{1}$ Anne Schohl, ${ }^{1}$ Dantong Jia, ${ }^{1}$ and ${ }^{\circ}$ Edward S. Ruthazer ${ }^{1}$ \\ ${ }^{1}$ Department of Neurology and Neurosurgery, Montreal Neurological Institute and ${ }^{2}$ Department of Psychiatry, McGill University, Montreal, Quebec H3A \\ 2B4, Canada
}

\begin{abstract}
Radial glia in the developing optic tectum extend highly dynamic filopodial protrusions within the tectal neuropil, the motility of which has previously been shown to be sensitive to neural activity and nitric oxide (NO) release. Using in vivo two-photon microscopy, we performed time-lapse imaging of radial glial cells and measured filopodial motility in the intact albino Xenopus laevis tadpole. Application of MK801 to block neuronal NMDA receptor (NMDAR) currents confirmed a significant reduction in radial glial filopodial motility. This reduction did not occur in glial cells expressing a dominant-negative form of cGMP-dependent protein kinase 1 (PKG1), and was prevented by elevation of cGMP levels with the phosphodiesterase type 5 inhibitor sildenafil. These results suggest that neuronal NMDAR activation results in the release of NO, which in turn modulates PKG1 activation in glial cells to control filopodial motility. We further showed that interfering with the function of the small GTPases Rac1 or RhoA, known to be regulated by PKG1 phosphorylation, decreased motility or eliminated filopodial processes respectively. These manipulations led to profound defects in excitatory synaptic development and maturation of neighboring neurons.
\end{abstract}

Key words: astrocyte; neuron-glia interaction; NMDA receptor; radial glia; retinotectal; synaptogenesis; PKG

\section{Significance Statement}

Radial glia in the developing brain extend motile filopodia from their primary stalk. Neuronal NMDA receptor activity controls glial motility through intercellular activation of cGMP-dependent protein kinase 1 (PKG1) signaling in glial cells. Manipulating PKG1, Rac1, or RhoA signaling in radial glia in vivo to eliminate glial filopodia or impair glial motility profoundly impacted synaptogenesis and circuit maturation.

\section{Introduction}

Glia cells perform many roles once attributed solely to neurons. It is now appreciated that glia significantly contribute to normal

\footnotetext{
Received Oct. 15, 2015; revised March 26, 2016; accepted March 30, 2016.

Author contributions: M.S., M.R.V.H., and E.S.R. designed research; M.S., M.R.V.H., A.S., and D.J. performed research; M.S., M.R.V.H., D.J., and E.S.R. analyzed data; M.S., M.R.V.H., and E.S.R. wrote the paper.

This work was supported by the Archimedes Foundation (M.S.), a McGill Faculty of Medicine Fellowship (M.S.), the McGill Neuroengineering Natural Sciences and Engineering Research Council of Canada (NSERC) Collaborative Research and Training Experience Training Program (M.S.), a Natural Sciences and Engineering Research Council of Canada Banting Postdoctoral Fellowship (M.R.V.H.), a Jeanne-Timmins-Costello Fellowship (M.R.V.H.), and a Canadian Institutes of Health Research operating grant (E.S.R.). E.S.R. has held salary awards from Fonds de researche du Québec, Canada Research Chairs, and the Killam Foundation. We thank S. Long, I. Hwang, and S. Chan for contributing to this study. The dn-PKG1 plasmid (G1alphaR-GFP) was a generous gift from D. Browning (Medical College of Georgia), and the dn-Rac1 (RacN17) and ca-RhoA (RhoAQ63L) were a gift from A. Fournier (McGill University).

The authors declare no competing financial interests.

Correspondence should be addressed to Edward S. Ruthazer, 3801 University St., McGill University, Montreal, QC H3A 2B4, Canada. E-mail: edward.ruthazer@mcgill.ca.
}

cognitive performance, and glial dysfunction has been noted in a number of psychiatric and neurological disorders (Prust et al., 2011; Van Horn et al., 2013; Verkhratsky and Parpura, 2016). Understanding how glia participate in the formation and maintenance of neural networks is crucial for better comprehension, prevention, and treatment of psychiatric and neurological disorders.

One aspect of glial behavior that has not yet been extensively explored in vivo is the fast movement of fine actin-based filopodial extensions formed by both astrocytes and radial glia. Astrocytic processes in situ are very dynamic (Haber et al., 2006; Bernardinelli et al., 2014). Interestingly, their motility is not random but is regulated by external effectors, such as glutamate, as 
demonstrated in astrocyte cell culture (Lavialle et al., 2011). Similar to astrocytic processes, filopodia of radial glial cells in the neuropil of developing Xenopus laevis brain are also very motile, extending, retracting, and restructuring over minutes in vivo (Tremblay et al., 2009).

Previous work from our laboratory demonstrated that the motility of fine filopodia of radial glia in the optic tectum of the tadpole is modulated by visual stimulation and reduced by blockade of neuronal NMDA-type glutamate receptors (Tremblay et al., 2009). Importantly, this study showed that radial glial cells in the Xenopus optic tectum do not express NMDA receptors (NMDARs), and therefore such changes in glial process dynamics with NMDAR blockade suggested the existence of intercellular signals that regulate glial motility in response to neuronal activity. Application of $\mathrm{N}^{\mathrm{G}}$-monomethyl-L-arginine to pharmacologically inhibit nitric oxide (NO) synthase was found to reduce radial glial motility, whereas the NO donor DetaNONOate restored normal motility under conditions of NMDAR blockade. Thus, the diffusible intercellular signaling molecule NO appears to function as a neuron-derived signal that regulates glial motility in response to sensory input and neural activity in the developing brain.

In the present study, using a combination of two-photon time-lapse imaging and whole-cell voltage-clamp recordings in the intact brain, we extend these findings by elucidating the signaling pathways downstream of neuronal NMDAR activation and $\mathrm{NO}$ that modulate radial glial filopodial motility in vivo. We show that neuronal activity-dependent regulation of glial filopodial motility is mediated by glial cGMP production, which in turn acts via cGMP-dependent protein kinase 1 (PKG1) to modulate cytoskeletal dynamics. We further demonstrate that radial glia filopodial motility can be regulated by the small GTPases Rac and RhoA. These manipulations that suppress radial glia filopodial dynamics significantly impair normal synaptic maturation of neurons in the developing visual system.

\section{Materials and Methods}

Animals. All animal experiments were approved by the Animal Care Committees of the Montreal Neurological Institute and McGill University, and were performed in accordance with the guidelines of the Canadian Council on Animal Care. Embryos of either sex were produced by human chorionic gonadotropin-induced natural breeding of adult albino Xenopus laevis frogs from our colony and maintained in $0.1 \times$ modified Barths solution with HEPES acid buffer (MBS-H). Animals were anesthetized for electroporation and injection procedures in $0.02 \%$ tricaine methanesulfonate (MS222) in $0.1 \times$ MBS- $\mathrm{H}$.

Electroporation and imaging. In vivo two-photon microscopy was performed with an Olympus FV300 confocal microscope custom-converted for multiphoton imaging and equipped with a MaiTai-BB Ti:sapphire femtosecond pulsed laser. Xenopus laevis tadpoles (stage 45) underwent tectal electroporation of a CMV promoter-driven plasmid [based on cloning vector pEGFP-C1 (Clontech)] for expressing membranetargeted farnesylated-enhanced green fluorescent protein (EGFPf). In some cases, EGFPf was coelectroporated with plasmid encoding one of the following: constitutively active small GTPase RhoAQ63L (ca-RhoA), dominant-negative small GTPase RacN17 (dn-Rac1), or dominantnegative PKG1 (dn-PKG1). Dn-PKG1 was expressed from the plasmid G1 $\alpha$ R-GFP, encoding only the regulatory domain of the PKG1 (Browning et al., 2001). RacN17 and RhoAQ63L constructs were inserted in the pcDNA expression vector. Imaging was performed $2 \mathrm{~d}$ later on stage 48 tadpoles.

Electroporation settings were modified as needed either to sparsely transfect individual electroporated radial glia or to drive expression in a large number of densely located radial glia in the tectum. DNA mixed with Fast Green dye for visualization was injected into the ventricle, platinum plate electrodes with a $3 \mu \mathrm{F}$ capacitor in parallel were placed on both sides of the tadpole's head, and three pulses of $37.5 \mathrm{~V}$ were delivered in both polarities. For sparse electroporation, pulse durations of $1.6 \mathrm{~ms}$ were used. For dense electroporation, each pulse was 3 $\mathrm{ms}$ in duration.

During imaging, tadpoles were immobilized by immersion in $0.2 \mathrm{~mm}$ pancuronium bromide in $0.1 \times$ MBS- $\mathrm{H}$ rearing solution. Tadpoles were placed in a form-fitting polydimethylsiloxane imaging chamber and held in place with a glass coverslip. Images were collected at $5 \mathrm{~min}$ time intervals on a custom-built two-photon microscope as $z$-stacks with $1 \mu \mathrm{m}$ steps. In the case of pharmacological treatments, the timeline depicted in Figure $2 C$ was followed. Two days after electroporation, three time-lapse images were taken at $5 \mathrm{~min}$ intervals followed by intraventricular injection of drugs (100 $\mu \mathrm{M}$ MK801, $5 \mu \mathrm{M}$ sildenafil, $100 \mu \mathrm{M}$ MK801 $+5 \mu \mathrm{M}$ sildenafil, or MBS-H control solution). After waiting $30 \mathrm{~min}$ for the drugs to take full effect, another series of three images was captured at 5 min intervals.

Motility quantification. Two-photon $z$-series images were deconvolved using AutoQuant software. Autofluorescent pigment cells and other cells expressing fluorescent protein were cropped out of $z$-stacks using ImageJ software. Maximum intensity projections of the stacks were created and an index of overall process motility was measured for each cell using a custom algorithm in Matlab (Sild et al., 2013). In brief, following Sobel edge detection, images were binarized and dilated using a six-pixel radius filter. Consecutive binarized and dilated images were subtracted to create difference images that reveal changing pixels over time. To highlight dynamic hotspots, difference images underwent $9 \times 9$ pixel boxcar filtering, and resulting images were multiplied by the original difference image to create the final pixel redistribution images. Motility index values were calculated per cell as the mean value of all nonzero pixels in the pixel redistribution images from three time points. This approach permits an unbiased index of motility to be calculated for each cell. It should be noted that this motility index is very sensitive to any image change and detects both biological and nonbiological (detector noise, photobleaching, etc.) components. We have previously reported (Sild et al., 2013) that even paraformaldehyde fixation only reduces the motility index by $\sim 40 \%$ due to these nonbiological factors. Therefore in healthy cells reproducible index changes of just a few percent reflect substantial changes in overall process motility.

Electrophysiology. Whole-cell patch-clamp recordings were made in the isolated intact brain from stage 47-48 albino Xenopus laevis tadpoles $2 \mathrm{~d}$ after dense coelectroporation of EGFPf with dn-PKG1, RhoAQ63L, or RacN17. Electroporation of EGFPf alone served as a control for the effects of electroporation. In preparation for recording, tadpoles were first anesthetized with MS222 $(0.02 \%$ in $0.1 \times$ MBS-H) and then placed in cold HEPES-buffered extracellular saline, which consisted of (in mM) the following: $115 \mathrm{NaCl}, 4 \mathrm{KCl}, 3 \mathrm{CaCl}_{2}, 3 \mathrm{MgCl}_{2}, 5$ HEPES, and 10 glucose, pH 7.2 (osmolarity: $\sim 250 \mathrm{mOsm}$ ). An incision was made along the dorsal midline of the optic tectum to expose the ventricular surface and the brain was dissected out and pinned to a block of Sylgard in a recording chamber with continuous perfusion of room temperature external saline. To gain access to tectal neurons, part of the ventricular membrane was carefully removed using a broken micropipette. Individual tectal neurons were visualized using an Olympus BX51 upright microscope with a $60 \times(0.8$ numerical aperture $)$ water-immersion objective and a CCD camera (Sony XC-75). Transfected radial glia cells were identified by epifluorescence excitation of EGFPf and tectal neurons in close proximity to the transfected radial glial cells were targeted for recordings.

Voltage-clamp recordings from tectal neurons were obtained using patch recording pipettes $(6-10 \mathrm{M} \Omega$ ) containing the following (in $\mathrm{mM}$ ): $90 \mathrm{CsMeSo}_{4}, 20$ HEPES, 20 tetraethylammonium, 10 EGTA, $5 \mathrm{MgCl}_{2}$, 2 ATP, 0.3 GTP, pH 7.20 (osmolarity: $250 \mathrm{mOsm}$ ). Miniature EPSCs (mEPSCs) were recorded in the presence of the $\mathrm{GABA}_{\mathrm{A}}$ antagonist picrotoxin (PTX, $100 \mu \mathrm{M}$ ) and tetrodotoxin (TTX, $1 \mu \mathrm{M}$ ) at a holding potential of $-70 \mathrm{mV}$.

Electrophysiological recordings were made using an Axopatch 200B amplifier (Molecular Devices) and signals were digitized (Digidata 1220) and sampled at $10 \mathrm{kHz}$, filtered at $2 \mathrm{kHz}$, and stored for off-line analysis with 

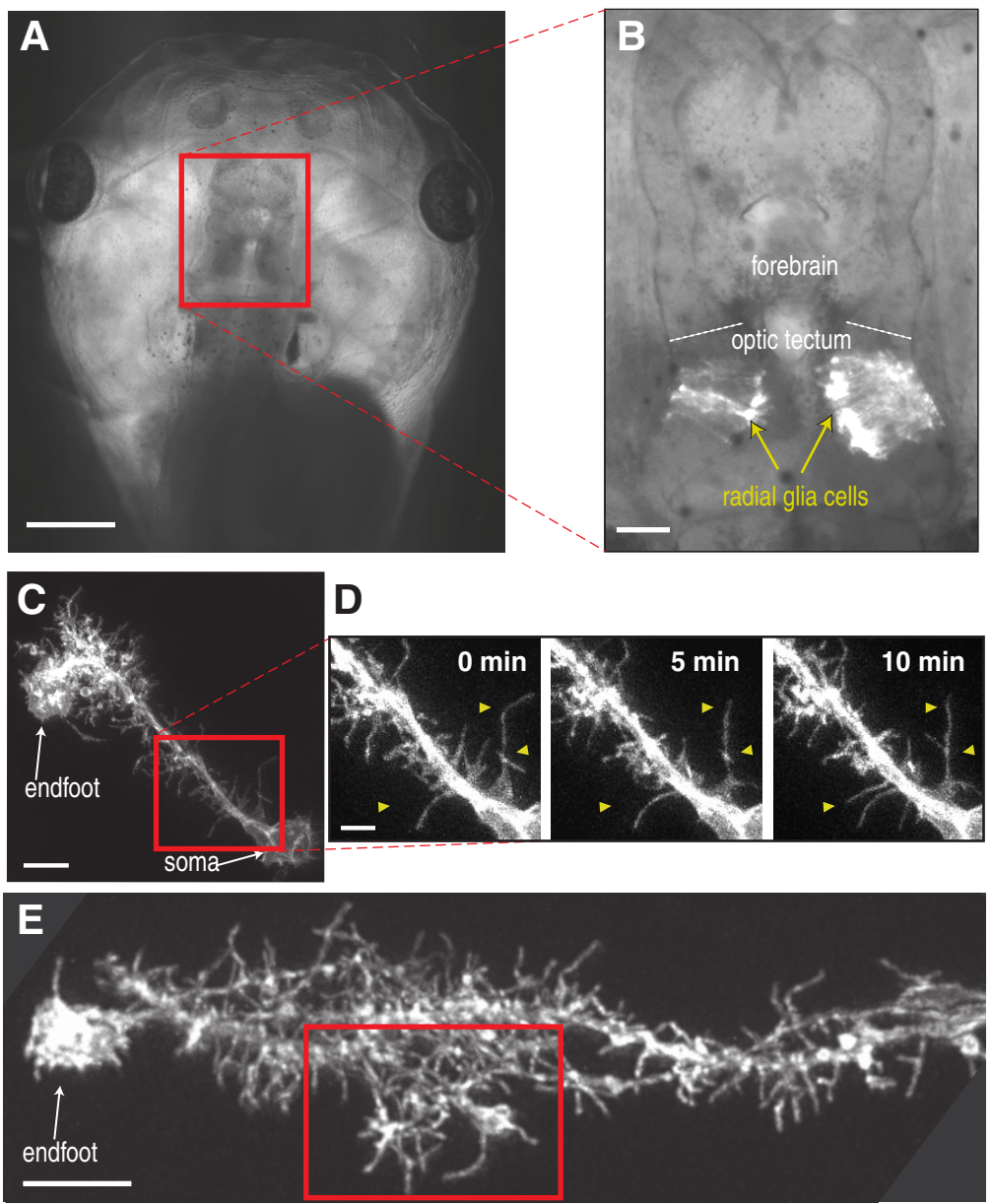

F Before MK801

H
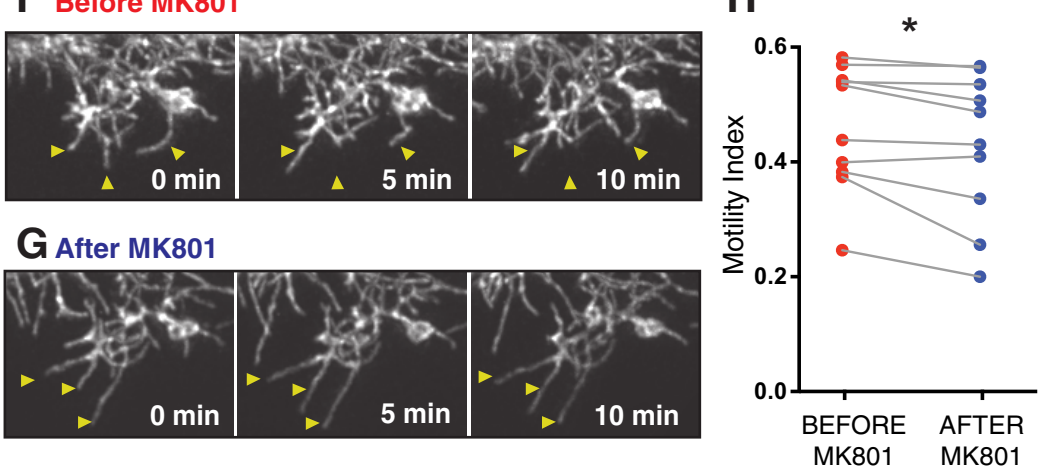

Figure 1. Radial glia exhibit filopodial process motility modulated by neural activity in vivo. $\boldsymbol{A}$, The entire brain is clearly visible in an intact stage 48 albino Xenopus laevis tadpole. $\boldsymbol{B}$, Dense electroporation of radial glial cells to express EGFPf in a portion of the optic tectum. Radial glial cell bodies appose the ventricular surface in the optic tectum and extend a long primary process tipped with one or more endfeet at the pial surface. C, A single radial glial cell visualized in vivo by two-photon microscopy expressing EGFPf. $\boldsymbol{D}$, Higher magnification of the rectangle in $\boldsymbol{C}$ shows spontaneous filopodial motility over $10 \mathrm{~min}$. Arrowheads highlight some remodeling processes. $\boldsymbol{E}$, Many fine filopodia extend into the tectal neuropil from the main process of a radial glial cell. $\boldsymbol{F}, \boldsymbol{G}$, The field within the rectangle is shown at multiple time points imaged before $(\boldsymbol{F})$ and $30 \mathrm{~min}$ after $(\boldsymbol{G})$ the application of MK801 $(100 \mu \mathrm{M})$. Arrowheads highlight examples of motile filopodia. $\boldsymbol{H}$, MK801 reduces the filopodial motility of radial glia. $N=10$ cells from 10 animals. ${ }^{*} p<0.05$, paired $t$ test. Scale bars: $\boldsymbol{A}, 500 \mu \mathrm{m} ; \boldsymbol{B}, 100 \mu \mathrm{m} ; \boldsymbol{C}, \boldsymbol{E}, 10 \mu \mathrm{m} ; \boldsymbol{D}, 5 \mu \mathrm{m}$.

pClamp 8 software. mEPSCs were analyzed using MiniAnalysis software (Synaptosoft), which automatically selects unitary events based on built-in a-amino-3-hydroxy-5-methyl-4-isoxazoleproprionic acid (AMPA) detection search criteria, using a $5 \mathrm{pA}$ amplitude threshold. All identified events were validated manually before calculating mean amplitude and frequency for each cell.

Anatomical synapse counting by immunohistochemistry. Animals were electroporated unilaterally in the optic tectum using settings to produce dense glial expression of EGFPf alone or together with one of the following plasmids: dn-PKG1, ca-RhoA, or dn-Racl. After 2 d, electroporated animals were fixed in $4 \%$ paraformaldehyde in PBS for $1 \mathrm{~h}$ at room temperature, transferred to ice-cold methanol, and postfixed overnight at $-20^{\circ} \mathrm{C}$. Animals were rehydrated in $75,50,25$, and $0 \%$ methanol in PBS and then washed $1 \mathrm{~h}$ in $100 \mathrm{~mm}$ Tris/ $\mathrm{HCl}$, $\mathrm{pH} 7.4,100 \mathrm{~mm} \mathrm{NaCl}$. Infiltration of the samples was done by incubation at room temperature overnight in $15 \%$ fish gelatin (Norland)/ $15 \%$ sucrose and $25 \%$ fish gelatin $/ 15 \%$ sucrose subsequently. Animals were then embedded and frozen in $20 \%$ fish gelatin/ $15 \%$ sucrose for cryosectioning. Sections were cut at $14 \mu \mathrm{m}$ thickness and exposed to antigen retrieval with $1 \%$ SDS in PBS for 3 min at room temperature. Sections were immunostained with rabbit anti-GluA1 (1:200; Abcam ab109450) and mouse anti-SV2 (1:1000; Developmental Studies Hybridoma Bank sv2-2a), with secondary antibodies Alexa-555 goat anti-rabbit IgG and Alexa-633 goat anti-mouse IgG, respectively. Digital $z$-stack images were taken on a Zeiss LSM 710 confocal microscope at $63 \times$ (Plan Apochromat, 1.4 N.A. oil-immersion objective). Sections positive for overexpressed EGFPf in the tectal radial glia were used for imaging. Per tectum, $2-3$ areas in the neuropil were selected and $z$-stacks were imaged with a step size of $0.37 \mu \mathrm{m}$ at $3 \times$ zoom. Since only one tectal lobe was electroporated per animal, 2-3 areas in the neuropil of the EGFPf-negative opposite hemispheres of each animal were imaged as internal controls.

For synapse quantification, three fields of 75 $\mu \mathrm{m}^{2}$ each were selected from each confocal $z$-stack (2-3 per hemisphere per animal) targeting zones of dense glial EGFPf expression. Analysis was performed on the third optical section beneath the cut surface of the histological section in fields devoid of vasculature or sectioning irregularities. Images were preprocessed with Fiji software using background subtraction (10-pixel-radius rolling ball) followed by median filtering ( 2 pixel radius). Images were then intensity thresholded using the Moments auto-thresholding function of Fiji. The Analyze Particles function was then used to identify puncta in the SV2 (presynaptic) and GluA1 (postsynaptic) channels, using a size criterion of $0.1-5.0 \mu \mathrm{m}^{2}$ area per punctum. "Synapses" were identified by taking the logical AND of these two channels to reveal overlapping pixels and the size criterion of $0.1-5.0$ $\mu \mathrm{m}^{2}$ area was again applied. This resulted in a conservative estimate of pre/postsynaptic juxtapositions. All analysis was performed blind to which plasmids had been electroporated.

Statistics. Statistical analyses were performed using Prism 6.0 (Graphpad). Data are expressed as mean \pm SEM. Post hoc analysis following ANOVA was performed using the Holm-Sidak test.

\section{Results}

Neuronal NMDARs regulate radial glial motility

The intact albino Xenopus laevis tadpole is translucent (Fig. 1A), facilitating live imaging of cells in the developing brain. Because 
the somata of radial glial cells in the optic tectum are directly apposed to the ventricular surface of the brain, these cells are efficiently transfected by passing current across the tectal lobes following intraventricular plasmid injection to electroporate DNA into the cells. Expression of membrane-targeted EGFPf permits complete visualization of transfected radial glial cells in the optic tectum from their periventricular somata to their pial endfeet (Fig. 1 $B, C$ ). Electroporation of DNA constructs using brief current pulses results in sparse transfection allowing individual radial glia to be observed in the intact animal (Fig. 1C). These cells have a characteristic bottlebrush morphology with extensive fine filopodial processes that exhibit a high degree of motility (Fig. $1 D)$, extending and retracting along the entire length of the cell, having their greatest density in the tectal neuropil toward the large pial endfoot.

Xenopus radial glia lack NMDARs (Tremblay et al., 2009). However, application of the NMDAR blocker MK801 (100 $\mu \mathrm{M})$ significantly reduced radial glial motility, presumably by acting on nearby neurons (Fig. 1E-G). Using an unbiased index of motility (Sild et al., 2013) to quantify the remodeling of filopodia on individual radial glial cells imaged by twophoton microscopy in intact animals, we observed a reduction in motility following 30 min exposure to MK801 in 9 of 10 cells (Fig. 1H).

\section{cGMP-dependent protein kinase in} radial glia acts downstream of neuronal NMDAR activation to regulate

filopodial dynamics

Previous work from our group indicated that NO production in response to neuronal NMDAR activation modulates activitydependent radial glial filopodial motility (Tremblay et al., 2009). An important downstream effector of NO signaling is the PKG1. Diffusible NO directly activates the soluble guanylate cyclase leading to elevated cGMP levels, which in turn stimulates PKG (Bredt and Snyder, 1989; Hobbs, 1997; Wang and Robinson, 1997). We therefore set out to test whether PKG in radial glial cells participates in the activity-dependent control of process motility.

Radial glia in the optic tectum were sparsely transfected to express either EGFPf alone or a dominant-negative construct consisting of the regulatory domain of PKG1 (dn-PKG1; Hou et al., 2004) along with EGFPf for imaging (Fig. $2 A, B$ ). To test the hypothesis that radial glial PKG1 activity downstream of neuronal NMDAR activation modulates glial motility, animals with dn-PKG1-expressing glia

C
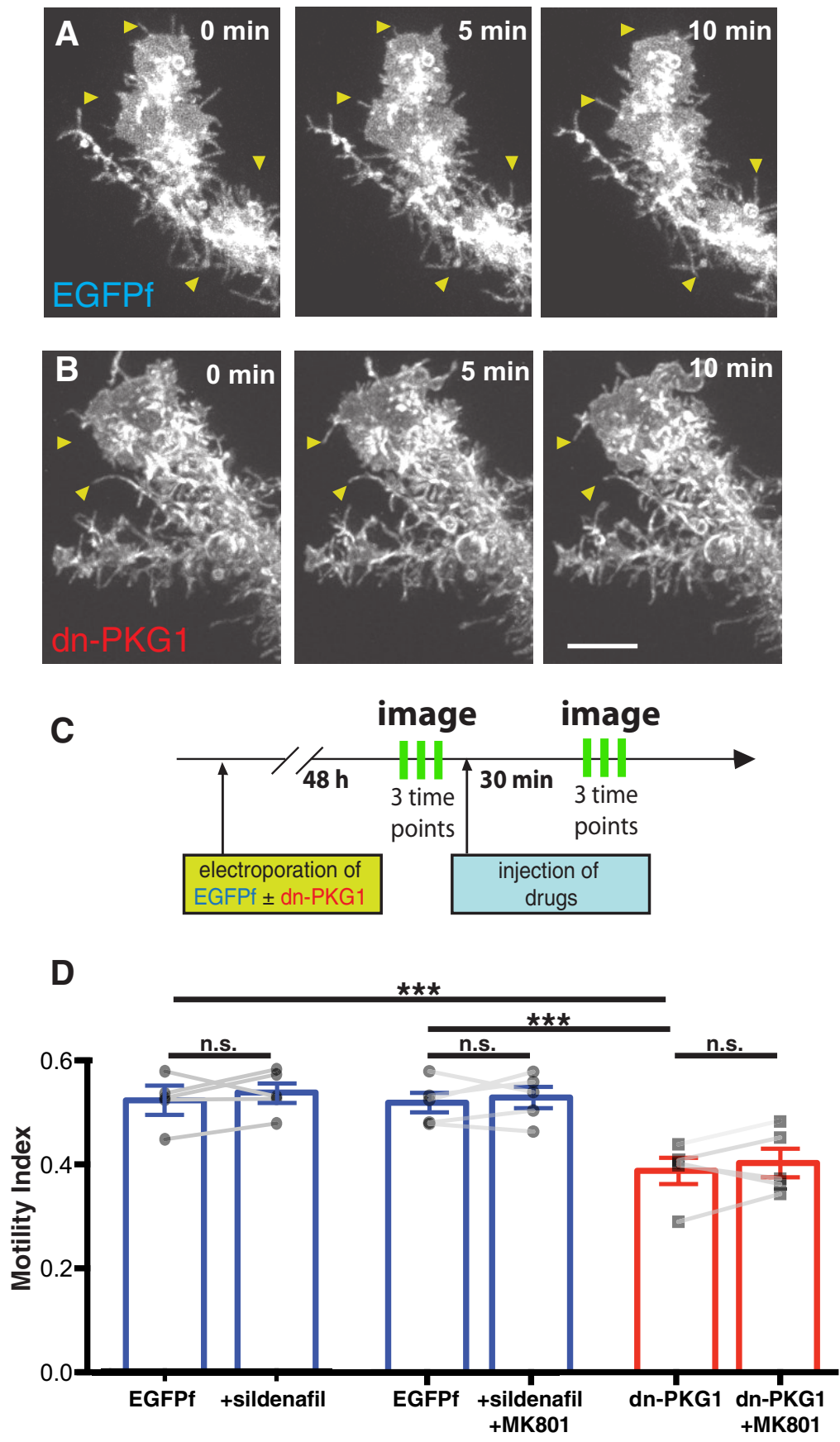

Figure 2. Neuronal NMDAR activity modulates radial glial filopodial motility through PKG1 signaling. $A, B$, Examples of in vivo time-lapse imaging of distal tips of EGFPf-expressing control $(\boldsymbol{A})$ and dn-PKG1-transfected $(\boldsymbol{B})$ radial glial cells imaged at 5 min intervals. Arrowheads highlight dynamic filopodia in $\boldsymbol{A}$ and reduced motility in dn-PKG1 cells in $\boldsymbol{B}$. C, Experimental protocol: radial glia were electroporated with EGFPf + dn-PKG1 or just EGFPf constructs. Two days after electroporation, radial glia were imaged in vivo three times at 5 min intervals, followed by injection of sildenafil $(5 \mu \mathrm{M})$, MK801 $(100 \mu \mathrm{M})$, or MK801 + sildenafil respectively into the tectal ventricle. After $30 \mathrm{~min}$, radial glia were imaged again three times every $5 \mathrm{~min}$. $\boldsymbol{D}$, Addition of sildenafil did not increase radial glial motility but the reduction in glial motility by tectal NMDAR blockade with MK801 (Fig. $1 \mathrm{H}$ ) was eliminated by inclusion of sildenafil to elevate PKG1 activity. Dn-PKG1-expressing radial glia exhibited significantly lower filopodial motility than EGFPf-expressing control cells. Dn-PKG1-expressing cells also did not respond to MK801 with further motility reduction, suggesting that glial PKG1 is downstream of neuronal NMDAR activation. $N=5$ cells from 5 animals for each condition. ${ }^{* *} p<0.005$, one-way ANOVA corrected for multiple comparisons; n.s., not significant by paired $t$ test. Scale bar, $10 \mu \mathrm{m}$. were treated with MK801. In parallel, EGFPf-expressing animals were injected intraventricularly with the phosphodiesterase type 5 inhibitor sildenafil citrate $(5 \mu \mathrm{M})$ to maintain elevated intracellular cGMP levels and therefore PKG1 activation. Some sildenafil-injected animals were treated with MK801 to deter- 
mine whether pharmacological activation of PKG1 could occlude the motility-reducing effects of NMDAR blockade. Radial glial motility was measured by collecting a time series of three twophoton images immediately before drug application, and once again $30 \mathrm{~min}$ after drug application (Fig. 2C).

Cells expressing dn-PKG1 exhibited significantly lower baseline (predrug) glial filopodial motility than the EGFPf cells (Fig. $2 D$ ), which is consistent with the idea that PKG1 controls glial dynamics. Sildenafil treatment of EGFPf-expressing cells to artificially increase PKG1 activity did not enhance glial motility, which is consistent with previous results suggesting that basal motility of radial glia in stage 48 tadpoles is close to maximal levels. In either sildenafil-treated or dn-PKG1-expressing radial glia, blockade of neuronal NMDARs with MK801 was no longer effective at reducing glial motility in contrast to MK801 alone (Fig. 1H). Together, these findings indicate that PKG1 in radial glial cells is an important effector for regulating glial process dynamics downstream of neuronal NMDAR activation.

\section{Radial glial filopodial motility decreases with age}

In the Xenopus optic tectum, developmental stages 45-48 represent a period of intensive synaptic remodeling with high rates of axonal and dendritic turnover. By stage 50, synapses in the retinotectal projection, while still undergoing gradual remodeling, are much more mature, in line with decreased rates of axonal and dendritic growth (Sakaguchi and Murphey, 1985; Wu et al., 1999; Akerman and Cline, 2006). Given the many lines of evidence for astrocytic involvement in synaptic development, including synapse ensheathing and contact-dependent signaling (for review, see Clarke and Barres, 2013), we wondered whether rates of radial glial filopodial motility might change over development as synapses become mature and stabilize. We therefore performed in vivo timelapse imaging of EGFPf-expressing radial glia in stage 48 and 50 animals. As described above, radial glia in stage 48 tadpoles extended many highly dynamic fine processes, including some long branches that elongated and retracted over minutes (Fig. $3 A$ ). In contrast, at stage 50, radial glia appeared "fuzzier", with a dense covering of fine processes that were less motile overall than in younger cells (Fig. 3B). The mean motility index in radial glia from stage 50 tadpoles was only $82.6 \pm 5.6 \%$ of the index measured in stage 48 animals (Fig. $3 C$ ). This age-related decrease in radial glial structural dynamics suggests a potential link between glial motility and synaptogenesis in the optic tectum. We therefore next examined how radial glial filopodial motility participates in synaptic development in the Xenopus optic tectum.

\section{PKG1 signaling in radial glia promotes normal synaptic development}

We performed dense electroporation to transfect multiple radial glia in the optic tectum with either dn-PKG1 or EGFPf as control, and $2 \mathrm{~d}$ later performed whole-cell patch-clamp electrophysiological recordings from tectal excitatory neurons surrounded either by EGFPf-transfected cells or by dn-PKG1-expressing glia with reduced filopodial dynamics (Fig. 4A). AMPAR-mediated mEPSCs were recorded in TTX and PTX, while holding the tectal neurons at $-70 \mathrm{mV}$ to block NMDAR currents (Fig. $4 B$ ). The mean amplitude of AMPA mEPSCs from neurons next to dnPKG1-expressing radial glia was indistinguishable from that recorded in control cells (Fig. 4C). However, mEPSC frequency was significantly reduced in the neurons neighboring dn-PKG1transfected radial glia (Fig. 4D), indicating a defect in excitatory synapse formation or maturation. Thus, the activity of PKG1 downstream of neuronal NMDAR activation not only signifi-
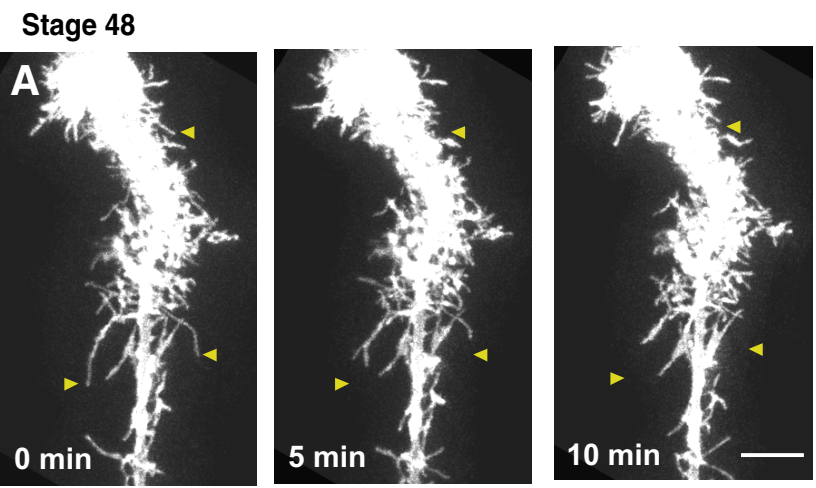

Stage 50
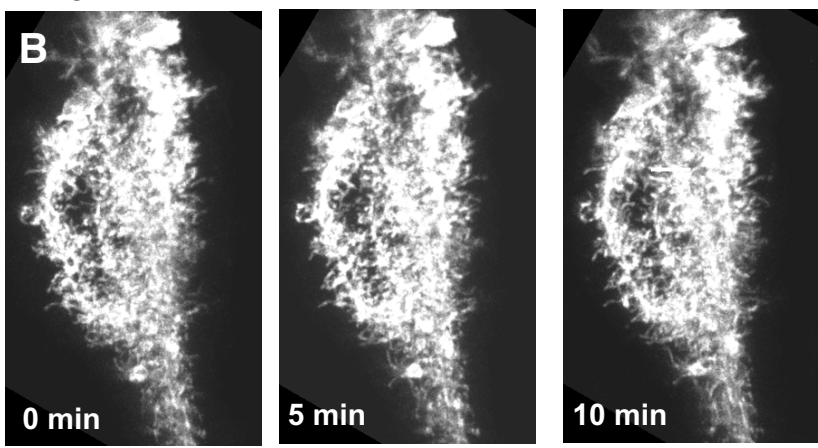

C
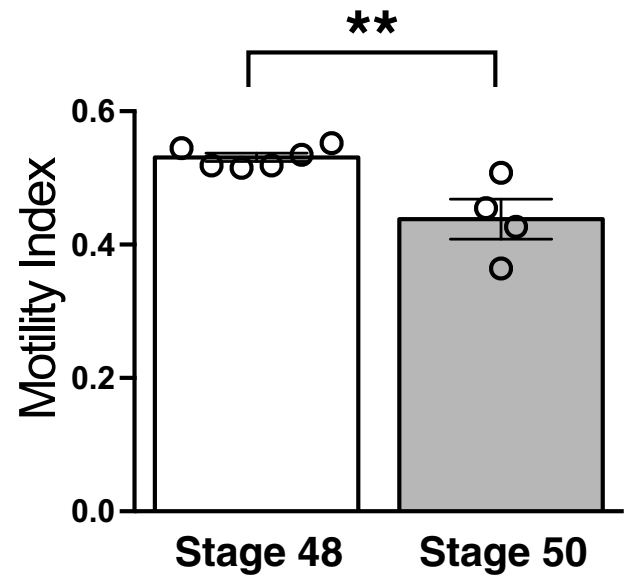

Figure 3. Radial glial filopodial motility decreases with age. $\boldsymbol{A}$, Time-lapse series of a radial glial cell in a stage 48 tadpole. $\boldsymbol{B}$, Time-lapse series of a radial glial cell in a stage 50 animal. Arrowheads illustrate examples of dynamic filopodia. All cells were electroporated at stage 45. C, Quantification of filopodial motility demonstrates that cells in older animals have reduced motility. Stage $48: N=6$ cells from 6 animals; stage $50: 4$ cells from 4 animals. ${ }^{* *} p<0.01$, Student's $t$ test. Scale bar, $10 \mu \mathrm{m}$.

cantly affects radial glial filopodial remodeling, but also appears to contribute to normal synaptic development in neighboring neurons, suggesting a potential contribution of glial motility to the process of circuit refinement. To determine whether these effects of PKG1 inhibition were likely to be due to their modulation of filopodial dynamics, we focused further attention on known cytoskeletal regulators downstream of PKG1 signaling.

Expressing constitutively active RhoA eliminates radial glial filopodia and prevents normal synaptic development RhoA and vasodilator-stimulated phosphoprotein, an activator of Rac1, are both phosphorylation targets of PKG (Smolenski et al., 1998; Ellerbroek et al., 2003; Hou et al., 2004). We therefore 

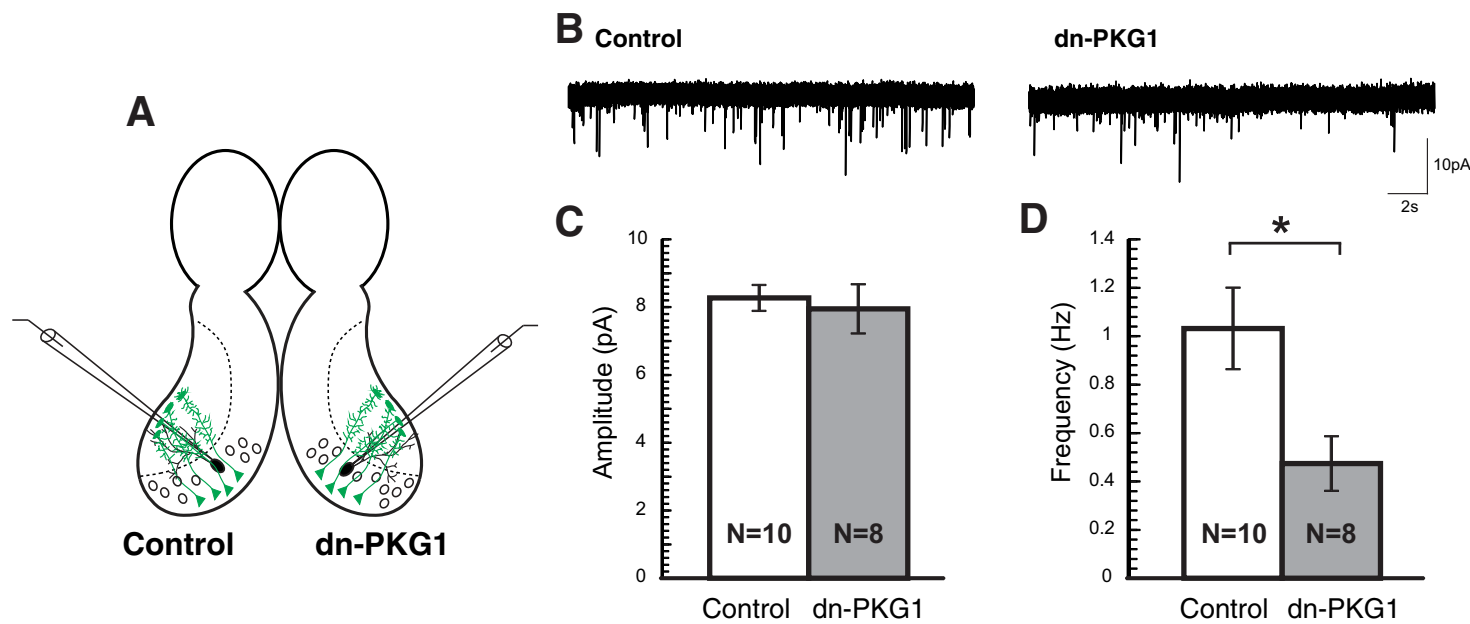

Figure 4. Expression of dn-PKG1 in radial glia lowers mEPSC frequency in adjacent neurons. $A$, Whole-cell patch-clamp recordings were performed on tectal neurons surrounded by radial glia expressing either EGFPf (left lobe on schematic) or dn-PKG1 (right lobe on schematic). $\boldsymbol{B}$, Example traces of AMPA mEPSC recordings from tectal neurons surrounded by EGFPf-transfected (control) or dn-PKG1-transfected radial glia. $\boldsymbol{C}, \boldsymbol{D}$, Neurons surrounded by dn-PKG1-expressing glia exhibit normal mEPSC amplitudes $(\boldsymbol{C})$ but significantly reduced frequencies (D). ${ }^{*} p<0.05$, Student's t test.

A

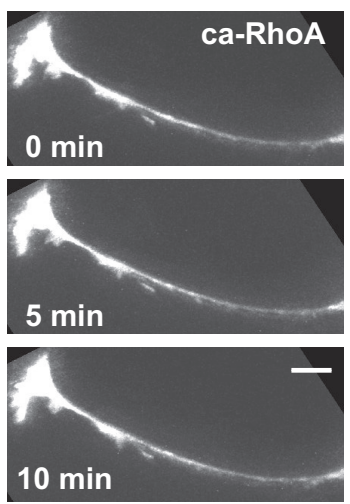

B

Control

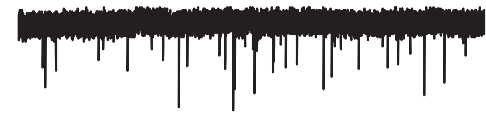

$\underset{1 \mathrm{~s}}{10 \mathrm{pA}}$

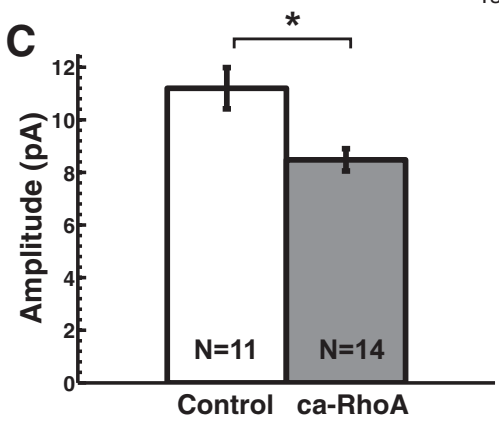

ca-RhoA
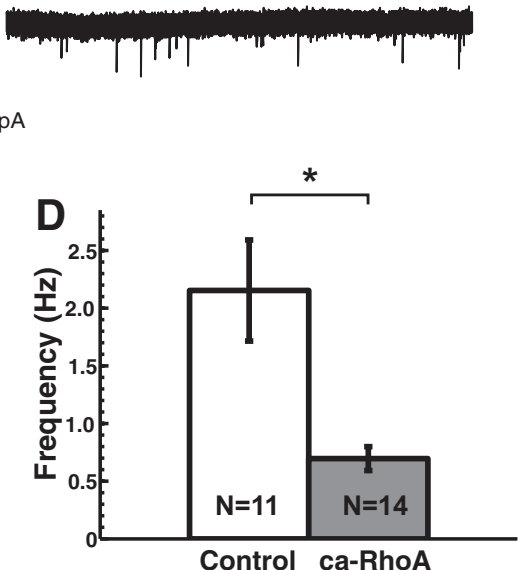

Figure 5. Expression of constitutively active RhoA eliminates most filopodial processes from radial glia and reduces mEPSC amplitude and frequency in nearby neurons. $A$, Example of a radial glial cell in vivo expressing ca-RhoA devoid of filopodia. Pial endfoot toward left. $\boldsymbol{B}$, Example traces of AMPA mEPSC recordings from tectal neurons surrounded by EGFPf-transfected (control) or ca-RhoA-transfected radial glia. $\boldsymbol{C}, \boldsymbol{D}$, Neurons surrounded by ca-RhoA-expressing glia exhibit significantly reduced mEPSC amplitude $(\boldsymbol{C})$ and frequency $(\boldsymbol{D}){ }^{*} p<0.05$, Student's $t$ test. Scale bar, $10 \mu \mathrm{m}$.

tested the roles of these small GTPases in radial glial dynamics and synaptic development. Expressing the RhoA(Q63L) constitutively active mutant form of RhoA (ca-RhoA) in radial glia had the dramatic effect of retracting nearly all of their filopodia (Fig. $5 A$ ). This extreme phenotype enabled us to ask whether radial glial filopodia are necessary for normal tectal excitatory synaptic development. We performed dense tectal electroporation to transfect radial glia with ca-RhoA and, $2 \mathrm{~d}$ after electroporation, recorded mEPSCs from tectal neurons surrounded by glia without filopodia (Fig. 5B). Mean AMPAR-mediated mEPSC frequency and amplitude were both significantly lower in brains transfected with ca-RhoA compared with those from EGFPftransfected control brains (Fig. 5C,D), suggesting a defect in excitatory synapse formation and maturation.

Dominant-negative Racl expression in radial glia suppresses filopodial motility and impairs synaptogenesis in neighboring neurons

In contrast to the devastating effects of ca-RhoA expression on glial morphology, cells expressing the Racl(N17) dominant- negative mutant of Racl (dn-Racl) maintained a relatively normal bushy appearance in static images (Fig. 6A). However, $\mathrm{dn}$-Racl expression caused a profound decrease in the rates of remodeling of glial filopodia, revealed by time-lapse imaging, effectively "freezing" the processes (Fig. 6B, C). Two days following dense electroporation of $\mathrm{dn}$-Racl into tectal radial glial cells, neurons surrounded by electroporated radial glia were targeted for whole-cell recording (Fig. 6D-F). Much like the dn-PKG1electroporated animals, and in contrast to ca-RhoA expression, mean mEPSC amplitude of unitary synaptic events was unaffected (Fig. 6E), while AMPAR-mediated mEPSC frequencies were significantly reduced compared with controls (Fig. $6 F$ ). A reduction in AMPA mEPSC frequency, which we observed whenever glial motility was impaired in our experiments, is often interpreted to indicate either a decrease in the total number of synapses formed or a failure of NMDAR-only "silent" synapses to traffic AMPARs to the synaptic cleft.

To distinguish between these possibilities, we performed immunohistochemical labeling of presynaptic (SV2) and postsynaptic (GluA1) markers on cryostat sections of optic tectum in 


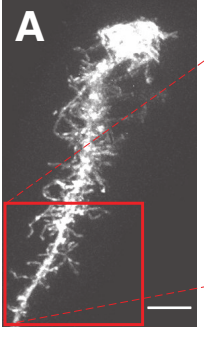

B

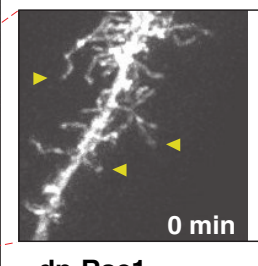

dn-Rac1

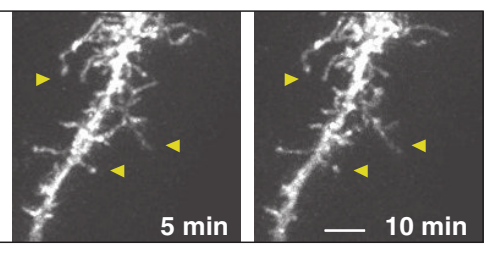

$5 \mathrm{~min}$

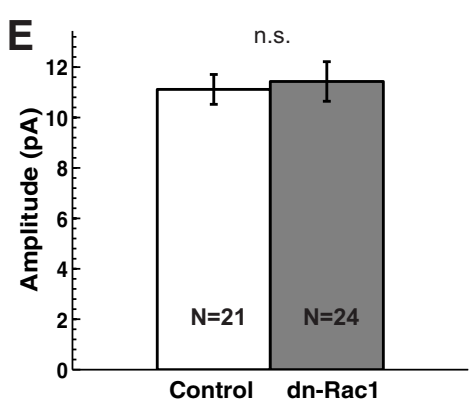

G

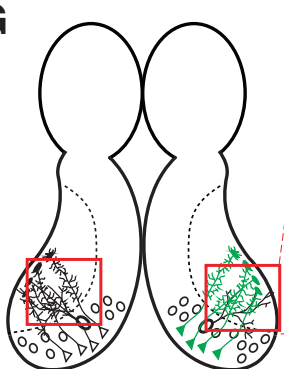

Untreated

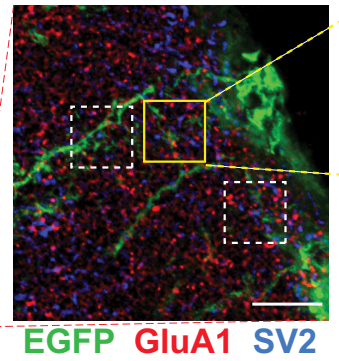

GluA1/SV2 colocalizations adjacent to transfected RG identified for blind counting using automated ImageJ macro
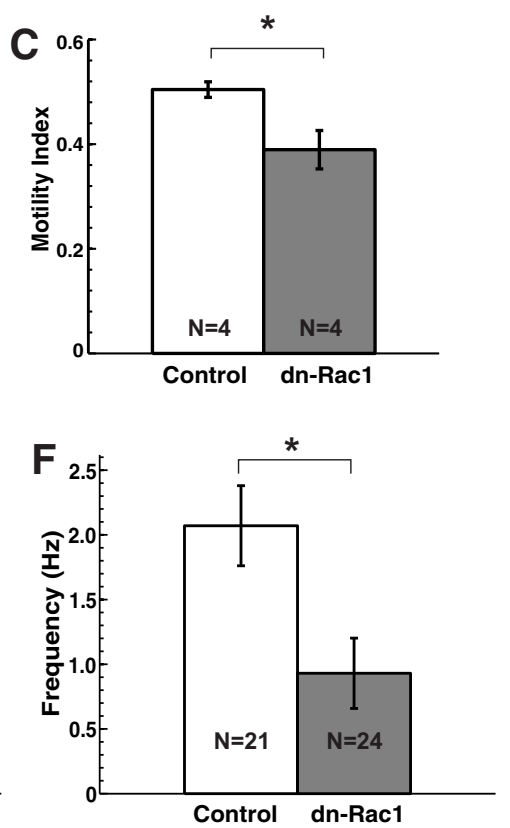

H

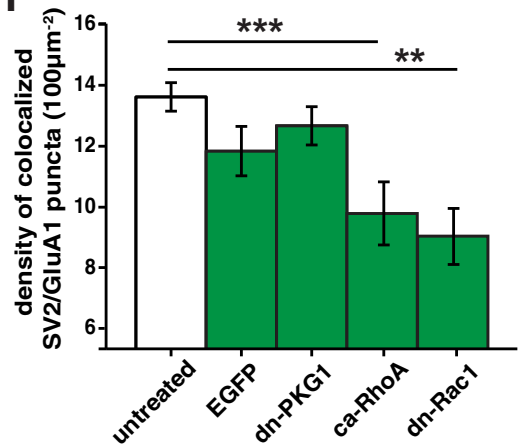

Figure 6. Expression of dominant-negative Rac1 suppresses radial glial filopodial motility and reduces synapse density in nearby neurons. $\boldsymbol{A}$ - $\boldsymbol{C}$, Example radial glial cell in vivo expressing dn-Rac1 is morphologically similar to a normal cell $(\boldsymbol{A})$ but exhibits greatly reduced filopodial motility $(\boldsymbol{B}, \boldsymbol{C})$. $\boldsymbol{D}$, Example traces of AMPA mEPSC recordings from tectal neurons surrounded by EGFPf-expressing (control) or dn-Rac1-transfected radial glia. $\boldsymbol{E}, \boldsymbol{F}$, Comparison of control neurons with neurons surrounded by dn-Rac1-expressing radial glia revealed no difference in mEPSC amplitude (E), but a profound decrease in mEPSC frequency $(\boldsymbol{F})$. G, Synapse density was measured by counting colocalized puncta of SV2 (presynaptic) and GluA1 (postsynaptic) immunofluorescence on brain sections in tectal neuropil regions electroporated to generate dense glial expression of EGFPf alone or with constructs that reduce glial motility. Untransfected opposite hemispheres were used as control. At least six fields per animal were analyzed from 25 animals. A sample field is shown before (top) and after (bottom) automated processing to identify "anatomical synapses" (white overlay). $\boldsymbol{H}$, Anatomical synapse density was significantly reduced in neuropil served by radial glia transfected with ca-RhoA ( $N=27$ fields from 4 tecta) or dn-Rac 1 ( $N=30$ fields from 4 tecta). Neither control EGFPf ( $N=45$ fields from 6 tecta) nor dn-PKG1 ( $N=78$ fields from 11 tecta) transfection decreased anatomical synapse density compared with untransfected hemispheres $(N=159$ fields from 25 tecta). ${ }^{*} p<0.05$, Student's $t$ test. ${ }^{* *} p<0.01,{ }^{* * *} p<0.005$, one-way ANOVA. Scale bars: $\boldsymbol{A}, \mathbf{G}, 10 \mu \mathrm{m} ; \boldsymbol{B}, 5 \mu \mathrm{m}$.

animals electroporated to drive dense local expression of EGFPf, either alone or together with dn-PKG1, ca-RhoA, or dn-Rac1 in the radial glia on one side of the brain (Fig. $6 G$ ). The untreated hemispheres served as internal controls. Confocal optical sections were analyzed at multiple locations in the tectal neuropil where the fine processes of EGFPf-expressing glial cells were richly ramified to measure the density of sites where the punctate labeling of presynaptic and postsynaptic markers was colocalized, indicating potential "anatomical synapses." Both ca-RhoA-expressing and dn-Racl-expressing animals exhibited significant reductions in anatomical synapse density near the transfected glial processes, while synapse number in the brains of animals electroporated with EGFPf alone was not significantly different from that in the untreated hemispheres (Fig. $6 H)$. Surprisingly, anatomical synapse density in dn-PKG1electroporated animals was not different from controls, despite the reduction in $\mathrm{mEPSC}$ frequency in neurons recorded from such animals. Thus, depending on the specific pathways affected and the degree to which glial processes are disrupted, the extent of circuit impairment can vary considerably, with manipulations like ca-RhoA expression that most severely affect glial morphogenesis, resulting both in reduced synapse density and function.

\section{Discussion}

Our results demonstrate that radial glial PKG1 mediates the activity-dependent regulation of radial glial filopodial motility downstream of neuronal NMDAR activation in the developing optic tectum as shown in Figure 7. We propose that NO released by neurons elevates glial cGMP, presumably through activation of the soluble guanylate cyclase, inducing glial PKG1 to modulate filopodial remodeling via Rho family small GTPases and their regulators, known to be targets for PKG phosphorylation. We further suggest that the dynamic remodeling of radial glial filopodia plays an important role in normal synapse formation and 


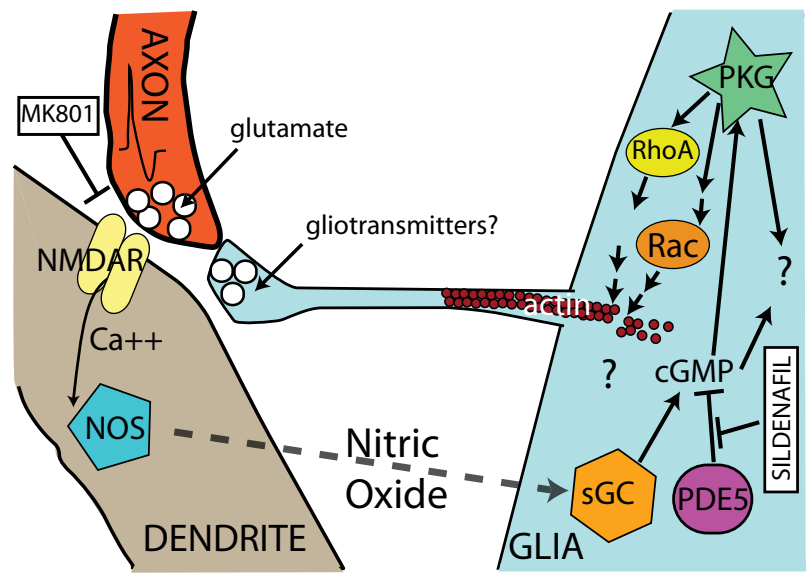

Figure 7. Model for neuronal NMDAR-dependent control of radial glial filopodial motility. Neuronal NMDAR activation leads to N0 production and diffusion to glia, where it stimulates the soluble guanylate cyclase ( $\mathrm{sGC}$ ) that elevates cGMP levels, activating PKG1. PKG1 regulates Rho family small GTPases, which control glial filopodial movement. Our data suggest that glial filopodial motility in turn participates in synapse formation and functional maturation.

maturation in vivo. Thus, radial glial cells both respond to and are able to influence neuronal activity in the developing brain, playing a critical role in normal development and function.

There are several possible ways by which glial filopodial motility might affect synaptic development. Astrocytes, a cell type related to radial glia, also have been shown to restructure their processes extensively in vitro (Hirrlinger et al., 2004; Haber et al., 2006). They too appear to regulate their morphology in an activity-dependent manner (Oliet et al., 2008; Bernardinelli et al., 2014), which may depend on glial PKG function (Borán and García, 2007). Astrocytes act as diffusion barriers to locally concentrate neurotransmitters and help target signaling molecules (Syková, 2001). They also aid in clearance of neurotransmitters, including glutamate and GABA, from the extracellular space (Schousboe, 2003; Schousboe and Waagepetersen, 2006), as well as accumulated ions, such as potassium (Chen et al., 1992). Furthermore, astrocytes metabolize glutamate and GABA for reuse by neurons, a function for which neurons themselves lack the necessary machinery (Bak et al., 2006). Astrocytes also actively release a variety of gliotransmitters that can contribute to synaptic function and plasticity (Volterra and Meldolesi, 2005; Haydon and Carmignoto, 2006; Araque et al., 2014). In addition to secreted signals, glial processes that extend toward neurons can activate contact-mediated signaling pathways, involving molecules implicated in synapse development, such as Ephs/ephrins (Murai et al., 2003; Filosa et al., 2009). Additionally, it has been shown that astrocytes express phagocytotic machinery that may participate in synapse elimination (Cahoy et al., 2008; Chung et al., 2013).

In contrast to astrocytes, radial glia have been much less studied in this context, with greater attention paid to their role as a neuronal and glial progenitor (Sharma and Cline, 2010; Dimou and Götz, 2014). In fish, frogs, and other lower vertebrates that exhibit extensive adult neurogenesis, and therefore maintain extensive radial glial populations throughout life, it is apparent that radial glia are capable of performing most if not all the functions attributed to astrocytes in mammals. We have previously shown that radial glia sense and respond to neuronal activity by modulating their filopodial motility (Tremblay et al., 2009). Immunohistochemical staining revealed that radial glial filopodia preferentially associate with synaptic terminals, indi- cating a potential function at synapses. A similar close interaction between synapses and a radial glia-like cell found in cerebellum, the Bergmann glia, has also been reported (Lippman et al., 2008). However, it was found that RhoG and Racl regulate Bergmann glia process length rather than motility, and during the period of cerebellar synaptogenesis in the mouse, RhoG and Rac1 dominant-negative expression in glia increased the total number of synapses, as detected by VGluT1 immunostaining (Lippman et al., 2008). In contrast, we found that manipulations that disrupted radial glial process extension and motility reduced, rather than increased, synapse number, as measured by a decrease in mEPSC frequency. Dn-Racl expression, which preserved but "froze" glial filopodia, affected only mean mEPSC frequency, and not amplitude. However, ca-RhoA expression, which practically eliminated glial fine processes, reduced both.

By measuring mEPSCs, we could obtain quantitative data on the number and properties of mature functional excitatory synapses that contain surface AMPARs, but an increased proportion of immature "silent" synapses lacking surface AMPARs would go undetected by this approach. We therefore also examined anatomical changes in synapse density in the neuropil surrounding transfected radial glia and found a decreased number of anatomical "synapses," as measured by colocalization of presynaptic SV2 and postsynaptic GluA1 immunofluorescence, after just $2 \mathrm{~d}$ of glial expression of dn-Racl or ca-RhoA. Expressing dn-PKG1 in glial cells also significantly reduced filopodial motility and mEPSC frequencies in adjacent neurons, but interestingly produced no change in anatomical synapse density. Compared to $\mathrm{dn}$-Rac1 expression, expression of dn-PKG1 results in a slightly less profound reduction in radial glial process motility, which may explain the ability of nearby synapses to form normally, despite reduced functional maturation. It is also likely that disruption of Rho family GTPase function in radial glia affects more than just structural motility, including receptor and transporter trafficking, vesicular release, and cell adhesion. Thus, inhibition of glial PKG1 signaling may constitute a more targeted disruption of neural activity-dependent glial dynamics, better revealing the specific consequences of uncoupling glial motility from neuronal NMDA receptor activation.

It is possible that D-serine or other glial-derived synapse maturation factors are released from glial processes and, therefore, as long as the filopodia are present, these factors may still be able to diffuse locally and partially influence neuronal development. The dynamic probing of filopodia may be important for more direct interactions with synapses either for uptake of neurotransmitters and ions, or for contact-mediated signaling, for example through Eph/ephrins or through targeted release of factors like D-serine, brain-derived neurotrophic factor, and thrombospondins. It is interesting to speculate that the relatively higher degree of glial process motility present in younger animals may constitute an efficient way for signaling between neurons and glia to occur while still preserving the topological capacity for extensive structural remodeling of developing axons and dendrites.

It will be important to determine whether the activitydependent movement of glial processes we observed has more of a random or directed nature. We have described a signaling pathway for activity-dependent regulation of overall glial motility downstream of neuronal NMDAR activation. However, glutamate is also known to induce astrocyte process motility in culture through glial mGluR5 and mGluR3 receptors (Lavialle et al., 2011). Intracellular $\mathrm{Ca}^{2+}$ transients and mGluR5 have also been linked to astroglial process dynamics in the mouse hippocampus with astrocytic mGluR5 activation inducing local calcium tran- 
sients that modulate synaptic transmission at CA1 pyramidal neuron synapses through gliotransmitter release (Panatier et al., 2011; Panatier and Robitaille, 2016). Local signaling by glutamate may provide a basis for directional motility of glial processes, perhaps guiding filopodia toward active synapses for better targeting of gliotransmission. Signaling mediated by rapidly diffusible NO, acting through glial PKG1, on the other hand, might regulate a more general, exploratory form of glial motility, which could help to detect partners for directional signaling. The respective roles of mGluR-dependent and $\mathrm{NO}$-dependent control of glial remodeling should be examined further by time-lapse imaging of glial filopodia in conjunction with uncaging or focal delivery of glutamate while disrupting various signaling mechanisms.

Synapses onto magnocellular neurons in the hypothalamus and pyramidal neurons in hippocampus and cortex have been shown to be actively ensheathed by astrocytic processes (Oliet et al., 2008; Bernardinelli et al., 2014). However, it remains unclear whether glial processes are actively attracted toward active synapses from a distance. Interestingly, increased astrocyte motility has been reported to facilitate dendritic spine outgrowth toward a source of glutamate in organotypic hippocampal slices as dendritic spines extend protrusions toward a source of glutamate (Verbich et al., 2012). Whether glutamate instructs the removal or attraction of glial membranes or whether it can do both depending on the context, remains to be elucidated.

Finally, it will be important to further elucidate the downstream actin-interacting effectors. Ezrin, a target of Rho kinase, has been shown to be expressed specifically in the fine actincontaining peripheral astrocyte processes, where it helps link F-actin to the cell membrane and contributes to process formation and movement (Lavialle et al., 2011).

\section{References}

Akerman CJ, Cline HT (2006) Depolarizing GABAergic conductances regulate the balance of excitation to inhibition in the developing retinotectal circuit in vivo. J Neurosci 26:5117-5130. CrossRef Medline

Araque A, Carmignoto G, Haydon PG, Oliet SH, Robitaille R, Volterra A (2014) Gliotransmitters travel in time and space. Neuron 81:728-739. CrossRef Medline

Bak LK, Schousboe A, Waagepetersen HS (2006) The glutamate/GABAglutamine cycle: aspects of transport, neurotransmitter homeostasis and ammonia transfer. J Neurochem 98:641-653. CrossRef Medline

Bernardinelli Y, Randall J, Janett E, Nikonenko I, König S, Jones EV, Flores CE, Murai KK, Bochet CG, Holtmaat A, Muller D (2014) Activitydependent structural plasticity of perisynaptic astrocytic domains promotes excitatory synapse stability. Curr Biol 24:1679-1688. CrossRef Medline

Borán MS, García A (2007) The cyclic GMP-protein kinase G pathway regulates cytoskeleton dynamics and motility in astrocytes. J Neurochem 102:216-230. CrossRef Medline

Bredt DS, Snyder SH (1989) Nitric oxide mediates glutamate-linked enhancement of cGMP levels in the cerebellum. Proc Natl Acad Sci U S A 86:9030-9033. CrossRef Medline

Browning DD, McShane M, Marty C, Ye RD (2001) Functional analysis of type lalpha cGMP-dependent protein kinase using green fluorescent fusion proteins. J Biol Chem 276:13039-13048. CrossRef Medline

Cahoy JD, Emery B, Kaushal A, Foo LC, Zamanian JL, Christopherson KS, Xing Y, Lubischer JL, Krieg PA, Krupenko SA, Thompson WJ, Barres BA (2008) A transcriptome database for astrocytes, neurons, and oligodendrocytes: a new resource for understanding brain development and function. J Neurosci 28:264-278. CrossRef Medline

Chen Y, McNeill JR, Hajek I, Hertz L (1992) Effect of vasopressin on brain swelling at the cellular level: do astrocytes exhibit a furosemidevasopressin-sensitive mechanism for volume regulation? Can J Physiol Pharmacol 70 [suppl]:S367-S373. Medline
Chung WS, Clarke LE, Wang GX, Stafford BK, Sher A, Chakraborty C, Joung J, Foo LC, Thompson A, Chen C, Smith SJ, Barres BA (2013) Astrocytes mediate synapse elimination through MEGF10 and MERTK pathways. Nature 504:394-400. CrossRef Medline

Clarke LE, Barres BA (2013) Emerging roles of astrocytes in neural circuit development. Nat Rev Neurosci 14:311-321. CrossRef Medline

Dimou L, Götz M (2014) Glial cells as progenitors and stem cells: new roles in the healthy and diseased brain. Physiol Rev 94:709-737. CrossRef Medline

Ellerbroek SM, Wennerberg K, Burridge K (2003) Serine phosphorylation negatively regulates RhoA in vivo. J Biol Chem 278:19023-19031. CrossRef Medline

Filosa A, Paixão S, Honsek SD, Carmona MA, Becker L, Feddersen B, Gaitanos L, Rudhard Y, Schoepfer R, Klopstock T, Kullander K, Rose CR, Pasquale EB, Klein R (2009) Neuron-glia communication via EphA4/ ephrin-A3 modulates LTP through glial glutamate transport. Nat Neurosci 12:1285-1292. CrossRef Medline

Haber M, Zhou L, Murai KK (2006) Cooperative astrocyte and dendritic spine dynamics at hippocampal excitatory synapses. J Neurosci 26:88818891. CrossRef Medline

Haydon PG, Carmignoto G (2006) Astrocyte control of synaptic transmission and neurovascular coupling. Physiol Rev 86:1009-1031. CrossRef Medline

Hirrlinger J, Hülsmann S, Kirchhoff F (2004) Astroglial processes show spontaneous motility at active synaptic terminals in situ. Eur J Neurosci 20:2235-2239. CrossRef Medline

Hobbs AJ (1997) Soluble guanylate cyclase: the forgotten sibling. Trends Pharmacol Sci 18:484-491. CrossRef Medline

Hou Y, Ye RD, Browning DD (2004) Activation of the small GTPase Racl by cGMP-dependent protein kinase. Cell Signal 16:1061-1069. CrossRef Medline

Lavialle M, Aumann G, Anlauf E, Pröls F, Arpin M, Derouiche A (2011) Structural plasticity of perisynaptic astrocyte processes involves ezrin and metabotropic glutamate receptors. Proc Natl Acad Sci U S A 108:1291512919. CrossRef Medline

Lippman JJ, Lordkipanidze T, Buell ME, Yoon SO, Dunaevsky A (2008) Morphogenesis and regulation of Bergmann glial processes during Purkinje cell dendritic spine ensheathment and synaptogenesis. Glia 56: 1463-1477. CrossRef Medline

Murai KK, Nguyen LN, Irie F, Yamaguchi Y, Pasquale EB (2003) Control of hippocampal dendritic spine morphology through ephrin-A3/EphA4 signaling. Nat Neurosci 6:153-160. CrossRef Medline

Oliet SH, Panatier A, Piet R, Mothet JP, Poulain DA, Theodosis DT (2008) Neuron-glia interactions in the rat supraoptic nucleus. Prog Brain Res 170:109-117. CrossRef Medline

Panatier A, Robitaille R. (2016) Astrocytic mGluR5s and the tripartite synapse. Neuroscience 323:29-34. CrossRef Medline

Panatier A, Vallée J, Haber M, Murai KK, Lacaille JC, Robitaille R (2011) Astrocytes are endogenous regulators of basal transmission at central synapses. Cell 146:785-798. CrossRef Medline

Prust M, Wang J, Morizono H, Messing A, Brenner M, Gordon E, Hartka T, Sokohl A, Schiffmann R, Gordish-Dressman H, Albin R, Amartino H, Brockman K, Dinopoulos A, Dotti MT, Fain D, Fernandez R, Ferreira J, Fleming J, Gill D (2011) GFAP mutations, age at onset, and clinical subtypes in Alexander disease. Neurology 77:1287-1294. CrossRef Medline

Sakaguchi DS, Murphey RK (1985) Map formation in the developing Xenopus retinotectal system: an examination of ganglion cell terminal arborizations. J Neurosci 5:3228-3245. Medline

Schousboe A (2003) Role of astrocytes in the maintenance and modulation of glutamatergic and GABAergic neurotransmission. Neurochem Res 28: 347-352. CrossRef Medline

Schousboe A, Waagepetersen HS (2006) Glial modulation of GABAergic and glutamat ergic neurotransmission. Curr Top Med Chem 6:929-934. CrossRef Medline

Sharma P, Cline HT (2010) Visual activity regulates neural progenitor cells in developing xenopus CNS through musashil. Neuron 68:442-455. CrossRef Medline

Sild M, Chatelain RP, Ruthazer ES (2013) Improved method for the quantification of motility in glia and other morphologically complex cells. Neural Plast 2013:853727. CrossRef Medline

Smolenski A, Bachmann C, Reinhard K, Hönig-Liedl P, Jarchau T, Ho- 
schuetzky H, Walter U (1998) Analysis and regulation of vasodilatorstimulated phosphoprotein serine 239 phosphorylation in vitro and in intact cells using a phosphospecific monoclonal antibody. J Biol Chem 273:20029-20035. CrossRef Medline

Syková E (2001) Glial diffusion barriers during aging and pathological states. Prog Brain Res 132:339-363. CrossRef Medline

Tremblay M, Fugère V, Tsui J, Schohl A, Tavakoli A, Travençolo BA, Costa Lda F, Ruthazer ES (2009) Regulation of radial glial motility by visual experience. J Neurosci 29:14066-14076. CrossRef Medline

Van Horn MR, Sild M, Ruthazer ES (2013) D-serine as a gliotransmitter and its roles in brain development and disease. Front Cell Neurosci 7:39. CrossRef Medline

Verbich D, Prenosil GA, Chang PK, Murai KK, McKinney RA (2012) Glial glutamate transport modulates dendritic spine head protrusions in the hippocampus. Glia 60:1067-1077. CrossRef Medline

Verkhratsky A, Parpura V (2016) Astrogliopathology in neurological, neurodevelopmental and psychiatric disorders. Neurobiol Dis 85:254-261. CrossRef Medline

Volterra A, Meldolesi J (2005) Astrocytes, from brain glue to communication elements: the revolution continues. Nat Rev Neurosci 6:626-640. CrossRef Medline

Wang X, Robinson PJ (1997) Cyclic GMP-dependent protein kinase and cellular signaling in the nervous system. J Neurochem 68:443-456. Medline

Wu GY, Zou DJ, Rajan I, Cline H (1999) Dendritic dynamics in vivo change during neuronal maturation. J Neurosci 19:4472-4483. Medline 